\title{
EIN FÜHRUNG
}

»Wenn die Bewässerung nicht zweckmäßig eingerichtet wird, ist es unmöglich aus dem Sandberg frische Wiesen und üppigen Wald hervorzuzaubern. "Diese Feststellung stammt nicht aus dem Mund eines Gartendenkmalpflegers im 21. Jahrhundert angesichts der zunehmend heißen und trockenen Sommer in Deutschland, sondern aus dem Jahr 1847 von dem berühmten Gartenarchitekten Hermann Fürst von Pückler-Muskau. Er sagte dies angesichts seines Besuchs im frisch angelegten Schlosspark Babelsberg. Erst mit der Einrichtung eines mit einer Dampfmaschine betriebenen Wasserwerks an der Glienicker Lake gelang es ihm, die umfangreichen Ländereien in einen üppigen Landschaftspark mit altem Baumbestand zu verwandeln.

Die Stiftung Preußische Schlösser und Gärten Berlin-Brandenburg (SPSG) verwaltet heute die malerisch gegenüber der Glienicker Brücke gelegene Babelsberger Gartenanlage. Angesichts der in den letzten Jahren auch regional immer deutlicher werdenden Anzeichen eines Klimawandels steht sie vor einer großen Herausforderung: Ihrer denkmalpflegerischen Aufgabe gemäß, muss sie die Anlage in ihrem künstlerischen Aussehen bewahren bzw. rekonstruieren.

Denn historische Gärten wie der Schlosspark Babelsberg weisen als Gesamtkunstwerke, in denen Natur, Kunst und Architektur in einem gestalterischen Einklang zu erleben sind, eine Besonderheit auf, die sie von anderen Kunstwerken unterscheidet: Sie bestehen hauptsächlich aus natürlichen und auf Witterungseinflüsse reagierenden Materialien: Bäumen, Sträuchern, Blumen, Rasen und Wiesen. Deshalb trifft der derzeitige Klimawandel mit seinen Auswirkungen diese Kunstwerke weitgehend ungeschützt.

Mit dem Klimawandel einhergehende Phänomene wie verlängerte Vegetationsperioden und zunehmende Trockenphasen im Frühjahr und Sommer führen dabei - wie im Schlosspark Babelsberg - zu neuen Problemen: So sind die Blühtermine nachweislich vorverlegt und machen die Pflanzen anfällig für Spätfrost; viele Bäume und Pflanzen sind dem

Im Text werden Maskulinum und Femininum verwendet, wenn es um Personen geht. Gemeint sind grundsätzlich alle Menschen, gleich welcher Geschlechtsidentität sie sich zugehörig fühlen. 
zunehmenden Trockenstress nicht gewachsen und werden anfälliger für - zum Teil neue Schädlinge oder sterben frühzeitig ab. Die in den letzten Jahren in rascher Folge auftretenden heftigen Stürme (zuletzt »Xavier« 2017) führten zu Windwürfen oder umfangreichem Baum- und Astbruch, auch weil die Wucht des Windes infolge der verlängerten Vegetationsperiode auf vollständig belaubte Kronendächer traf. Die kurzen Starkregen fließen zu großen Teilen oberflächlich ab, füllen die Wasserspeicher im Boden nicht mehr auf und waschen die nur mit hohem ökonomischem Aufwand wiederherzustellenden alten Wegesysteme regelmäßig aus.

In der schon im 18. Jahrhundert bei Gartenliebhabern berühmten Wörlitzer Gartenanlage in Sachsen-Anhalt, einem der frühesten Landschaftsparks nach englischem Vorbild auf dem europäischen Festland, kämpfen die Gartenverantwortlichen seit Jahrhunderten mit einem ganz anderen Problem - dem Zuviel an Wasser. Seit den sogenannten Jahrhundertfluten, den extremen Hochwassern im Sommer 2002 und 2013, ist die zerstörerische Kraft des Wassers auch der Öffentlichkeit bewusst. Ein aufwendiges wissenschaftliches Projekt der Kulturstiftung Dessau-Wörlitz untersuchte den Zustand des gesamten Gehölzbestands, den Nährstoffgehalt der Böden sowie die Grundwasserdynamik der Gartenanlage, um daraus die erforderlichen Pflegemaßnahmen ableiten zu können. Möglicherweise sind solche Modellprojekte richtungsweisend für die Bewahrung des wertvollen Baum- und Pflanzenbestands in den zahlreichen denkmalgeschützten historischen Gärten Deutschlands.

Neben dem Schlosspark Babelsberg und dem Wörlitzer Park in Sachsen-Anhalt, die beide zum Weltkulturerbe der UNESCO zählen, waren der Große Tiergarten in Berlin und der im Südosten Brandenburgs in Cottbus gelegene Fürst-Pückler-Park Branitz Gegenstand der Untersuchungen ${ }^{1}$ der interdisziplinären Arbeitsgruppe (IAG) »Historische Gärten im Klimawandel« der Berlin-Brandenburgischen Akademie der Wissenschaften.

In ihrer dreijährigen Laufzeit (2016-2019) hat die IAG versucht, Antworten auf die Frage zu finden, ob und wie historische Gärten, Parkanlagen und Kulturlandschaften als geschützte Kulturgüter unter den veränderten klimatischen Bedingungen zukünftig fachgerecht bewahrt werden können und wie der denkmalpflegerische Auftrag entsprechend erfüllt werden kann. Sie griff damit Forderungen der »Erklärung von Sanssouci zum Erhalt der historischen Gärten und Kulturlandschaften «² vom 5. September 2014 auf: Dort wurde konstatiert, dass es einen »akuten und mittelfristigen Forschungsbedarf « gibt, um »Gefährdungen durch die Folgen des Klimawandels für die historischen Gärten, Bauwerke und Kulturlandschaften« adäquat beschreiben zu können. Das - so der Wortlaut der Erklä-

1 Vgl. für die kunsthistorische Beschreibung der von der IAG für ihre Untersuchungen ausgewählten Gärten den Beitrag von Adrian von Buttlar in diesem Band.

2 https://www.spsg.de/index.php?id=10752. Die »Erklärung von Sanssouci« ist im Zuge des internationalen Kongresses »Historische Gärten im Klimawandel « im September 2014 verfasst worden. Vgl.: Generaldirektion der Stiftung Preußische Schlösser und Gärten Berlin-Brandenburg (SPSG, Hg.) (2014): Historische Gärten im Klimawandel. Empfehlungen zur Bewahrung. Leipzig: Seemann Henschel. 
rung - »ist eine gemeinsame Aufgabe für Natur- und Geisteswissenschaften, denn nur so lassen sich längerfristig wirksame Handlungsstrategien entwickeln, um den negativen Auswirkungen auf unser kulturelles Erbe nachhaltig und grenzüberschreitend zu begegnen.«

Mit dem vorliegenden Band präsentiert die interdisziplinäre Arbeitsgruppe die Ergebnisse ihrer Arbeit. Die Forschungen zu den vier ausgewählten Beispielgärten erfolgte in den folgenden thematischen Clustern: "Kunstgeschichte und Gartendenkmalpflege«, »Naturräumliche Ausstattung « und »Gesellschaftliche Rahmenbedingungen und sozialwissenschaftliche Perspektiven . $^{3}$

Die im Kapitel »Kunstgeschichte und Gartendenkmalpflege« versammelten Beiträge nehmen die (kunst-)historischen und denkmalpflegerischen Grundlagen der historischen Gärten als schöpferische Ergebnisse der Gartenkunst in den Blick und befassen sich unter anderem mit der Entwicklung der Gartendenkmalpflege in theoretischer und praktischer Hinsicht.

In dem Kapitel »Naturräumliche Ausstattung« wurden von den Autoren relevante Informationen und Untersuchungsergebnisse zur naturräumlichen Ausstattung der vier Beispielgärten erstmals systematisch zusammengestellt und vergleichend bewertet, um zu erkennen, welche Risiken und Veränderungen aus dem Klimawandel resultieren und welche Rolle hierbei standörtliche Faktoren spielen. Ausgangspunkt der Untersuchungen war die Annahme, dass extreme Klimabedingungen bereits bei der Entstehung dieser Gärten eine Rolle gespielt haben, da diese allesamt im kontinental geprägten Teil Ostdeutschlands liegen, aber durch die spezifischen Standortverhältnisse in ganz unterschiedlicher Weise auf die von jeher geringen Jahresniederschläge und auf die anhaltenden sommerlichen Trockenperioden reagieren.

Nicht nur die physische Umwelt, sondern auch gesellschaftliche Bedingungen spielen für eine Bewahrung historischer Gärten eine Rolle. Denn Gärten werden von Menschen genutzt, geschätzt und verwaltet und sind somit genuin soziale Institutionen. Die Autoren des Kapitels »Gesellschaftliche Rahmenbedingungen und sozialwissenschaftliche Perspektiven « vermitteln ein facettenreiches Bild derselben und zeigen erstmals die Bedeutung sozialwissenschaftlicher Analysen für ein ganzheitliches Bild historischer Gärten unter den Bedingungen des Klimawandels auf.

Um der Tatsache gerecht zu werden, dass es sich beim Klimawandel um ein globales Phänomen handelt, hat die interdisziplinäre Arbeitsgruppe »Historische Gärten im Klimawandel « zwei international ausgerichtete Tagungen durchgeführt. Die daraus hervorgegangenen Beiträge, die sich auf historische Gärten in Großbritannien, Italien und Russland beziehen, sind im Kapitel »Internationale Perspektiven« versammelt.

»Theoria cum praxi« war die Losung von Gottfried Wilhelm Leibniz, dem Begründer der Berliner Akademie der Wissenschaften. Dass diese Losung gerade mit Bezug auf den

3 Vgl. jeweils ausführlich die Kapiteleinleitungen auf den S. 3-5, 151-153, 271-272 und 335-336. 
zukünftigen Erhalt der historischen Gärten unter den Bedingungen des Klimawandels mehr denn je seine Berechtigung hat, zeigt das Abschlusskapitel des Bandes: Nur durch eine systematische interdisziplinäre Erforschung sämtlicher Rahmenbedingungen und Gegebenheiten sowohl aus aktueller als auch historischer Perspektive kann das erforderliche Wissen generiert werden, das die mit dem Erhalt der Gärten befassten Akteure benötigen, um die entsprechenden (praktischen) Maßnahmen ergreifen zu können. 